\title{
Compressibility and Crushability Reproduction through an Amorphous Haptic Interface
}

\author{
Amir Berrezag, Yon Visell, and Vincent Hayward \\ UPMC Univ. Paris 6, ISIR, Institut des Systèmes Intelligents et de Robotique, 75005, \\ Paris, France \\ $\{$ amir.berrezag, yon.visell, vincent.hayward\}@isir.upmc.fr \\ http://www.isir.upmc.fr
}

\begin{abstract}
The demonstration involves a new haptic interface based on a wide-bandwidth electromagnetic valve and a pair of chambers filled with a magnetorheological fluid. It is intended to reproduce, with highfidelity, properties of complex materials that are experienced through pressing and squeezing actions, at the detriment of shape.
\end{abstract}

\section{Concept}

By a large margin, the greatest majority of haptic interfaces $[1,2,3]$, make contact with their users through hard surfaces. Exceptions include devices based on vibrators stitched into garments [4], or airborne energy transport [5]. In everyday life, however, haptic interaction often takes the form of squeezing objects to inspect their internal state and structure (e.g., "what's the pressure in my bike tires", compressibility; or "how much coffee is left in the bag?", crushability; or "how fresh is that bread?", compressibility and crushability), or to provoke a mechanical outcome (e.g., to extract a liquid or a paste, to displace grains) that depends crucially on the sensory feedback resulting from a squeezing action between fingers or a compressing action against a hard surface.

The mechanical responses resulting from such actions, often include oscillatory components that can be modeled through deterministic or stochastic techniques, in combination with a separable, low frequency component $[6,7]$. While the reproduction of the oscillatory components alone via hard surfaces may be sufficient to determine vivid perceptual outcomes $[8,9]$, another haptic simulation approach, proposed here, involves the artificial reproduction of the sensory experiences resulting from squeezing actions performed via a deformable haptic interface. This can reproduce a range of mechanical responses, e.g. viscosity and elastic deformation for the low frequency components, and micro-damage, micro-collisions, bubble dynamics, turbulence, etc., from high-frequency components.

We present a haptic interface comprising two deformable chambers (of arbitrary shape) which, crucially, are made of an inextensible sheet, preferably of oriented polymers, such as BoPP (biaxially-oriented polypropylene, frequently utilized in food packaging. The chambers are connected by a conduit and filled with magnetorheological (MR) fluid. The conduit is locally constricted to a channel passing between the polar pieces of the magnetic circuit of an electromagnet, 
see Fig 1. This system operates as a high-bandwidth valve that can modulate the flow in the whole frequency range relevant to haptic and tactile interaction. The system further includes a differential pressure sensor that enables closed-loop haptic simulation, that is, modulation of the mechanical response (compressibility of the chamber) as a function of the electromagnet input. In this sense, the system is 'passive' and cannot 'create' mechanical energy. It can only restitute supplied energy, linearly as well nonlinearly. The system can reproduce the compressibility behavior of everyday objects, yet is 'amorphous' since it is incapable of reproducing shape, which is, instead, determined by the user's grip. The electromagnetic actuator readily responds at driving signal frequencies up to $1 \mathrm{kHz}$, effectively covering the entire range of frequencies of mechanical stimuli that the tactile sense is sensitive to. The device is driven by an audio-bandwidth voltage amplifier. Large magnitude variations in the compressibility are possible, since the actuator can be driven with high power (up to a few dozen watts), with the heat readily dissipated by the MR fluid.

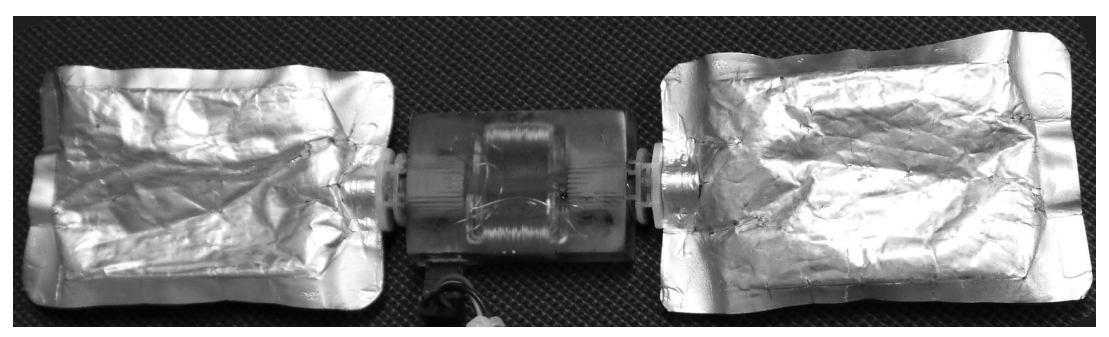

Fig. 1. Haptic interface comprising two squeezable bags, an electromagnetic valve, and a differential pressure sensor

\section{Demonstration}

We will demonstrate that such an amorphous interface can reproduce viscous, textural, and flow effects that are typical of haptic rendering. This device could be used in standalone mode, integrated in a product interface (soft button, slider or switch), or integrated in the graspable manipulandum of a force feedback device. Some advantages of this technique include its utility for simulating the variable compressibility of soft bodies or textures during direct, distributed contact with the skin using a single voltage control input to an electromagnet. Potential applications for the rendering of haptic effects in virtual environments or other human-machine interfaces are self-evident, e.g., gaming or rehabilitation.

One method of interacting with the device is illustrated in Fig. 2. When the user compresses one pouch, the MR fluid tends to flow to the other through the valve. A voltage-controlled magnetic field created in the circuit changes the viscosity of the liquid causing a variation of pressure. The high-frequency components in the excitation cause the pressure to vary rapidly in the entire system since pressure waves propagate at very high celerities in liquids. The hands can 
feel vibrations that be used to stimulate different textures and material behavior. The response is of very high fidelity owing to the absence of transmission modes in a viscous incompressible fluid medium.

Other configurations are also possible - for example, with one of the chambers made of an elastic material instead of inextensible plastic sheet. The advantage would be a persistent returning force from the elastic bag that would function to return the liquid to the grip (the non-extensible chamber) so that the hand can feel the stimuli either when compressing the bag and when releasing it. It is also possible to interact with the interface by holding it vertically with one hand and letting the fliud migrate from the upper chamber to the lower one through gravity. The potential energy expended during this process is amply sufficient to provoke a strong sensory experience. Another configuration involves a pair of chambers, one being large and elastic and the other small and stiff, with the two placed in mutual contact. When such an assembly is squeezed in the hand or in between the fingers, the fluid tends to flow through from the smaller bag to the larger owing to hydrostatic laws. Other modes of interaction may involve pushing against a hard surface (under the foot for example) or coupling two squeezing partners, the later potentially giving rise to unusual games and interaction paradigms due to to an uncanny, bilateral sensorimotor coupling.

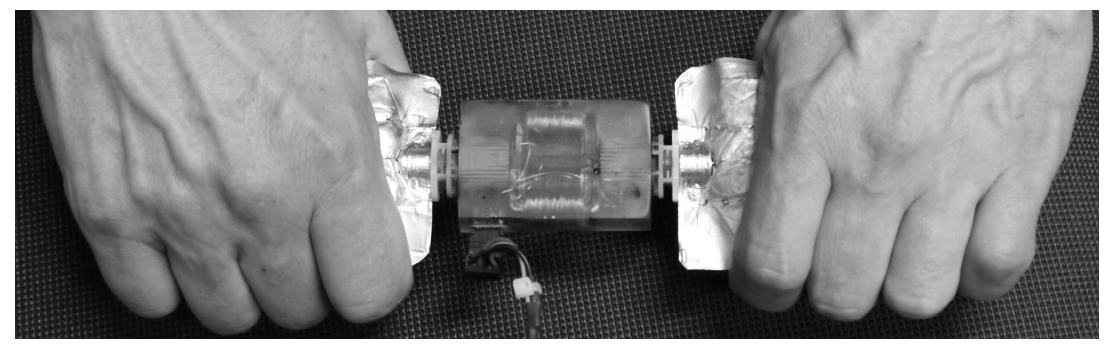

Fig. 2. One of many modes of interaction

\section{Rendering and Evaluation}

The amorphous, fluid-based device is capable of displaying compressabilities that vary at a rate of approximately $1 \mathrm{kHz}$. We explored its application to the simulation of virtual quasi-amorphous objects, such as viscous fluids in an elastic sack (e.g., a water balloon or silicone breast implant) or granular media (e.g., a sack of ground coffee, dried lentils). Such effects are accomplished by varying the rate, $f$, at which fluid can flow through the valve, thereby the compressibility $C=d V / d p$, where $d V$ is a differential increment of change in volume and $d p$ is an increment of change in differential pressure. Changes in the mean flow rate are effected by selecting the DC component of the actuator driving signal, and correspond to modifying the apparent viscosity of fluid in the chambers, while variations in the transitory components of the actuator driving signal yield 
transitory variations in the compressibility associated with texture-like effects. The latter are analogous to rendering textures via high-frequency variations in the viscous damping of a force-feedback interface [10].

We conducted a preliminary evaluation to collect some information about how users would describe these novel stimuli. In a first experiment, users were presented with twelve different combinations of transitory signal textures and viscous compressabilities, and were asked to describe what they felt using a few words. Four participants were recruited for this evaluation, all of them members of the authors' institute. The stimuli were synthesized with an actuator voltage signal of the form $s(t)=A_{1} p(\lambda, t)+A_{2} n\left(f_{c}, t\right)+A_{3} s_{0}$, where $A_{1}, A_{2}, A_{3}$ are amplitudes, $s_{0}$ is a DC driving signal voltage, $p(\lambda, t)$ is Poisson (shot) noise with parameter $\lambda$, and $n\left(f_{c}, t\right)$ is lowpass filtered white noise (cutoff frequency $f_{c}$ ). The Poisson noise source provided a "granular" texture component, the lowpass noise source provided a "frictional" roughness, and the DC component modulated mean (bulk) compressibility, producing a viscosity-like effect. Twelve stimuli were synthesized by selecting parameters $\lambda, f_{c}, A_{1}, A_{2}, A_{3}$ by hand. Participants pressed on one of the fluid-filled membranes until it was emptied and reported in one to two phrases what they felt. The responses were heterogeneous, with the most common label applied by participants referring to "vibrations" and "viscosity", perhaps indicating that the texture and viscous configurations were clearly felt. Other common responses referred to material properties/interactions including: "friction noise", "air bubbles", "granular", and "elasticity".

In a second experiment using the same twelve stimuli, we selected six labels ("Texture", "Vibrations", "Friction/sliding", "Stiffness/elasticity", "Granular", "Viscosity") that were commonly supplied during the first experiment and asked twelve participants to select one or two of the labels that best described each stimulus. The response rates differed significantly among the labels (Anova 1way, $p<0.01$ ), with the most common choices being Viscosity (mentioned in $64 \%$ of responses) and Granular (58\%) and Vibrations (56\%). "Texture" (23\%) was selected least often. Although these results provide some indication of the most prominent effects that can be achieved and the range of potential associations that can be evoked, a more systematic investigation will be required to explore the potential phenomenology of the device, as well as the psychophysics underlying how particular virtual material attributes may be perceived [11].

\section{References}

1. Hayward, V., MacLean, K.E.: Do it yourself haptics, part-I. IEEE Robotics and Automation Magazine 14(4), 88-104 (2007)

2. Benali Khoudja, M., Hafez, M., Alexandre, J.-M., Kheddar, A.: Tactile interfaces: a state of the art survey. In: International Symposium on Robotics, Paris, pp. 721-726 (March 2004)

3. Visell, Y.: Tactile sensory substitution: Models for enaction in HCI. Interacting with Computers 21(1-2), 38-53 (2009)

4. Gunther, E., O'Modhrain: Cutaneous grooves: Composing for the sense of touc. Journal of New Music Research 32(4), 369-381 (2003) 
5. Hoshi, T., Iwamoto, T., Shinoda, H.: Non-contact tactile sensation synthesized by ultrasound transducers. In: Proceedings of the World Haptics Conference, pp. 256-260 (2009)

6. Turchet, L., Nordahl, R., Serafin, S., Berrezag, A., Dimitrov, S., Hayward, V.: Audio-haptic physically-based simulation of walking on different grounds. In: Proceedings of the 2010 the IEEE International Worshop on Multimedia Signal Processing, MMSP 2010, pp. 269-273 (2010)

7. Visell, Y., Fontana, F.: Walking with the Senses: Non-visual perceptual techniques for walking in simulated environments. Logos Verlag (2012)

8. Wiertlewski, M., Lozada, J., Hayward, V.: The spatial spectrum of tangential skin displacement can encode tactual texture. IEEE Transactions on Robotics 27(3), 461-472 (2011)

9. Visell, Y., Giordano, B., Millet, G., Cooperstock, J.: Vibration influences haptic perception of surface compliance during walking. PLoS ONE 6(3), e17697 (2011)

10. Campion, G., Gosline, A.H., Hayward, V.: Passive viscous haptic textures. In: Proceedings from Symposium on Haptic Interfaces for Virtual Environment and Teleoperator Systems, pp. 379-380 (2008)

11. Bergmann-Tiest, W.M., Kappers, A.M.L.: Analysis of haptic perception of materials by multidimensional scaling and physical measurements of roughness and compressibility. Acta Psychologica 121(1), 1-20 (2006) 